\title{
Interoperability Testing Based on a Fault Model for a System of Communicating FSMs
}

\author{
Vadim Trenkaev*, Myungchul Kim, and Soonuk Seol \\ Information and Communications University \\ 58-4 Hwaam-Dong, Yuseong-Gu, Daejon, 305-732, Korea \\ vad@elefot.tsu.ru, \{mckim, suseol\}@icu.ac.kr
}

\begin{abstract}
This paper presents a fault model for interoperability testing of communication protocols that are modeled by communicating finite state machines, and proposes a technique that extends an initial interoperability test suite, which is given by another existing method, to be a test suite that can detect "almost all" interaction faults based on the fault model. We start with an interoperability test suite derived by a known method and develop a technique for the fault coverage analysis and a technique for the extension of the test suite in order to achieve high fault coverage. We illustrate the proposed techniques with TCP protocol. The fault coverage analysis concludes that the test suite has $100 \%$ fault coverage with respect to the proposed fault domain and does not need to be extended. It is shown that our method is applicable to practical protocols and can be used to make interoperability test suites have high fault-detecting capability.
\end{abstract}

\section{Introduction}

In the field of communication protocols testing there exist a number of types of testing, in particular, conformance and interoperability testing. Conformance testing is used when a single protocol entity is tested. The objective of conformance testing is to establish whether an implementation under test conforms to its protocol specification. Interoperability testing is used for checking if two or more protocol entities can operate as a system. The objective of interoperability testing is to check whether two or more implementations that usually pass the conformance testing can interact and if so whether they communicate with each other correctly providing the expected services.

In the case of conformance testing, there are many research works in which test suite derivation methods are based on a formal fault model [1]. A Labeled Transition System (LTS) and a Finite State Machine (FSM) can serve as examples of a formal model of the system that consists of a single entity. To evaluate the quality of a test suite, two well-known criteria are used: a fault coverage and total length of a test suite, i.e., a high-quality test suite must detect all or almost all faults of a given fault

\footnotetext{
On leave from Tomsk State University 
domain and must be short enough. The fault domain including such types of faults as any output and/or transfer fault in the implementation at hand is widely used.

Here we notice in the case of interoperability testing, we need a more complex formal model of a system under test (SUT), for example a system of communicating finite state machines (SCFSM). There are a number of papers that deal with testing a SCFSM [2-5] with respect to a formal fault model [6]. In this paper, we use this model for interoperability testing. Namely, we assume that a joint behavior of the protocol entities is described by a SCFSM and at most one component FSM can be faulty. To be more rigorous, the following is assumed. Communicating FSMs have been thoroughly tested in isolation and found conforming to their specifications, and interact through a media that can be modeled by an FSM with a single state. However, the component FSMs can be incompatible. For example, some options are implemented only for a single component FSM; the component implementations can have different codes for the same variable. Such faults can be modeled as output faults of component FSMs, i.e., when the output of an appropriate transition is wrong. Thus we consider interoperability faults to be output faults in component implementations. It is assumed that the access to internal interfaces of protocol entities is not granted.

In this paper, we develop a technique for a test suite derivation w.r.t. single output faults of component transitions which are responsible for interacting between communicating FSMs. The technique includes the fault coverage analysis of a given interoperability test suite is derived by another existing method and an algorithm for extending the initial test suite to increase its fault coverage.

We illustrate the proposed fault coverage analysis with TCP protocol, using the initial interoperability test suite which is derived by the method from [8]. The test suite for the TCP has $100 \%$ fault coverage w.r.t. the above fault domain.

The rest of the paper is structured as follows. Section 2 presents the related work while preliminaries are given in Section 3. In Section 4, we present a fault model for interoperability testing. We explain in Section 5 how a given test suite can be expanded in order to have a higher fault coverage and propose a technique for such extension. In Section 6, we apply the proposed fault coverage analysis to the TCP protocol. Section 7 concludes the work.

\section{Related Work and Motivation}

There are a number of papers that deal with interoperability testing [7-12]. In most of them when deriving an interoperability test suite, the technique is based on a reachability analysis. Depending on how the protocol implementations to be tested interact, and depending on the points of observation and control, different interoperability testing architectures are used. Some papers present a framework for interoperability testing $[9,10]$. Viho et al [10] show how the existing concepts of conformance testing can be used for interoperability testing. Griffeth et al [12] present a method for automatic generation of test cases which cover all the required interoperations and contain a number of test cases with total length close to minimum. We also notice that vari- 
ous kinds of interactions can occur between protocol entities; the latter can communicate synchronously or asynchronously, based on the single or multi stimulus principles [11].

However, the authors of most existing papers do not discuss the fault coverage of an interoperability test suite. The goal of interoperability testing often is to execute all possible system interactions not caring which interoperability faults can be detected. Therefore, the fault coverage of a derived interoperability test suite remains unknown, i.e., we usually can say nothing about faults that are detected by the test suite. To discuss the fault coverage we need to formally describe the set of faults to be detected. The fault coverage of a given test suite is then calculated as the ratio of number of faults that are detected by the test suite, to total number of possible faults. The fault coverage shows the quality of a test suite in terms of its fault-detecting capabilities; the higher is the fault coverage the higher is the quality of a test suite [13]. Thus, the evaluation of the fault coverage of a given test suite is an important issue in the protocol testing. In this paper we propose a model of interoperability faults and a method of the calculation of the fault coverage.

\section{Preliminaries}

\subsection{Finite State Machine}

We define a deterministic FSM $A$ as a 6-tuple $\left(S, X, Y, \psi, \varphi, s_{0}\right)$, where $S$ is a finite set of states with $s_{0}$ as the initial state, $X$ is a finite set of inputs, $Y$ is a finite set of outputs, $\psi: S \times X \rightarrow S$ is the transition function and $\varphi: S \times X \rightarrow Y$ is the output function. The 4-tuple ( $\left.s, x, y, s^{\prime}\right), s, s^{\prime} \in S, x \in X, y \in Y$, is called a transition, where $s, s^{\prime}$ are, respectively, the initial and final states of the transition, and $x, y$ are, respectively, the input and the output symbols.

We assume that a FSM has a reset capability, i.e., there is a special reset input " $r$ " that takes the FSM from any state to the initial state. The output "null" is produced when performing this transition. Moreover, we assume that the transition $(s, r$, null, $\left.s_{0}\right), s \in S$, is always correctly implemented.

In this paper we use only complete FSMs. However, usually a protocol specification is not completely specified. For each state and each undefined input at the state, we augment the description of an FSM by use of a completeness assumption [1]. The corresponding complete FSM remains at the state while producing external output "null". In a real situation the "null" output can be detected by waiting a specified time-out period.

As usual, we extend the output function to input sequences. We assign $\psi(s, \varepsilon)=s$ and $\varphi(s, \varepsilon)=\varepsilon$, for any $s \in S$, where $\varepsilon$ is so-called empty symbol. Given states $s, s^{\prime} \in S$, input sequence $\alpha=x_{1} x_{2} \ldots x_{k} \in X^{*}$, and output sequence $\beta=y_{1} y_{2} \ldots y_{k} \in Y^{*}, \varphi(s, \alpha)=\beta$ if there exist states $s_{1}=s, s_{2}, \ldots, s_{k}, s_{k+1}=s^{\prime}$ such that $\psi\left(s_{i}, x_{i}\right)=s_{i+1}, \varphi\left(s_{i}, x_{i}\right)=y_{i}$, $i=1, \ldots, k$. 
The equivalence relation between two states $s$ and $s^{\prime}$ of FSM $A$ holds if $\forall \alpha \in X^{*}$ $\left[\varphi(s, \alpha)=\varphi\left(s^{\prime}, \alpha\right)\right]$, otherwise, the states are non-equivalent. Two FSMs $A$ and $B$ are said to be equivalent if their initial states are equivalent; this means that the machines have one and the same behavior. For non-equivalent FSMs there is a sequence such that the responses of the machines do not coincide. A sequence is said to distinguish FSM $A$ and FSM $B$ if FSMs have different output responses to this sequence.

Let $X=X_{1} \cup X_{2} \cup \ldots \cup X_{n}$. Given a sequence $\alpha \in X^{*}$ and $k=1, \ldots, n$, let a sequence $\beta \in X_{k}^{*}$, is obtained from the sequence $\alpha$ by deleting all the symbols that are not in $X_{k}$. Then the sequence $\beta$ is called $X_{k}$-projection of sequence $\alpha$.

\subsection{System of Communicating Finite State Machines}

Formal methods can be applied for interoperability testing if a joint behavior of the protocol entities is formally described. In this paper we assume a behavior of the specification system and of a system under test is described by a system of communicating finite state machines [2] that exchange symbols over channels. Each protocol entity is a FSM (further a component machine) while each channel is a perfect bounded FIFO (first-in and first-out) queue.

We assume a SCFSM works in a slow environment [2], i.e., the next input can be applied only when all the processes in the system under the previous input have been completed. Neither the specification system nor its implementation has a live-lock, i.e., component machines execute finite number of transitions under any external input. Each component machine has no input queue and produces a pair of outputs, an external output and an internal output, as a response to a current input.

Let a system under test be specified as a system of communicating finite state machines $A 1=\left(Q, X_{1} \cup E_{2}, Y_{1} \times E_{1}, \psi_{1}, \varphi_{1}, q_{0}\right)$ and $A 2=\left(T, X_{2} \cup E_{1}, Y_{2} \times E_{2}, \psi_{2}, \varphi_{2}, t_{0}\right)$ with the structure shown in Fig. 1.

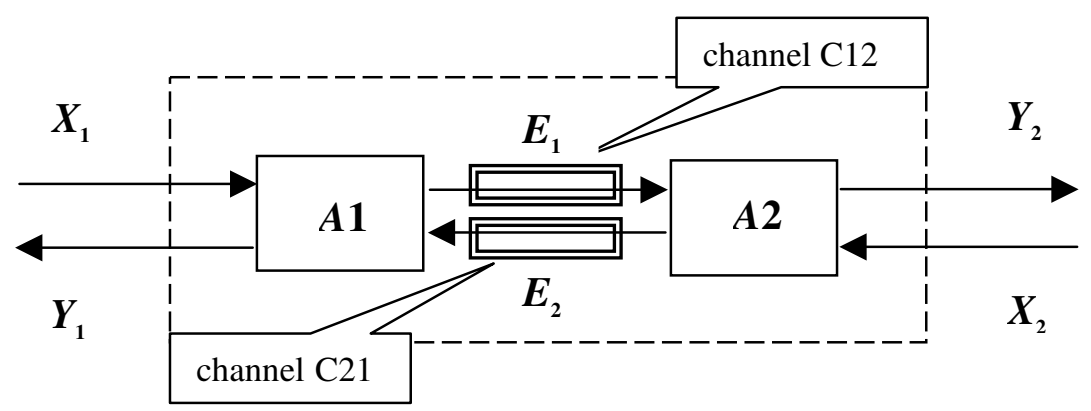

Fig. 1. System under test as system of communicating finite state machines.

In order to deal with the situation when only one output is produced by a component machine we assume that each alphabet $Y_{1}$ and $E_{1}\left(Y_{2}\right.$ and $\left.E_{2}\right)$ has empty symbol $\varepsilon$. Thus output pair $(e, \varepsilon)$ or $(\varepsilon, y), e \in E_{1}, y \in Y_{1}$, corresponds the situation when a compo- 
nent machine produces output $e$ or $y$, respectively. Thus the SCFSM has $X=X_{1} \cup X_{2}$ as the set of external inputs, $Y=Y_{1} \cup Y_{2}$ as the set of external outputs, while $E_{1}$ and $E_{2}$ are internal alphabets. For the sake of simplicity, the alphabets are assumed to be pairwise disjoint. Note, that formally we ought to consider $Y=Y_{1} \times Y_{2}$ as external output alphabet. However, since there exist only external pairs $(\varepsilon, y)$ or $(y, \varepsilon)$ then we omit empty symbol $\varepsilon$ and consider $Y=Y_{1} \cup Y_{2}$.

The channel $\mathrm{C} 12$ (respectively $\mathrm{C} 21$ ) is a perfect FIFO queue linking from the FSM $A 1$ to the FSM $A 2$ (from the FSM $A 2$ to the FSM $A 1$, respectively). The content of the channel $\mathrm{C} 12$ (respectively $\mathrm{C} 21$ ) is a sequence of symbols of $E 1$ (respectively $E 2$ ). Note, for the above assumptions the content of a channel is a symbol, i.e., the channel size is one.

We use 4-tuple $\left(q, t, c_{12}, c_{21}\right)$ to represent a global state of the SCFSM, where $q \in Q$, $t \in T, c_{12}$ and $c_{21}$ are contents of channels $\mathrm{C} 12$ and $\mathrm{C} 21$, respectively. In other words, a global state of the SCFSM consists of a pair of states of the component machines and the content of each channel. A global state is called stable if all channel queues are empty. Otherwise, it is called transient. Due to the principle of a slow environment, a stimulus from the environment can be submitted only when the system is at a stable state.

A joint behavior of communicating FSMs $A 1$ and $A 2$ can be represented by means of a reachability graph that is similar to a global state machine [2] and a composition of FSMs [5]. The reachability graph $G$ is pair $(V, E)$, where the set $V$ of vertices represents the set of global states of the system. The set of edges $E$ corresponds to transitions of the system from one global state to another. Each edge is labeled by an input/output pair of an appropriate component machine. The initial vertex is the stable state $\left(q_{0}, t_{0}, \mathcal{E}, \mathcal{E}\right)$.

The reachability graph is constructed by simulation of a behavior of the system under external inputs, i.e., is derived as follows:

- Let $g \in G$ be a current vertex representing a stable state $(q, t, \mathcal{\varepsilon}, \mathcal{E})$ while $x \in X_{1}$ being an external input, i.e., the FSM $A 1$ has a stimulus from environment. Then there is an outgoing edge from the vertex $g$ labeled by pair $x / \alpha, \alpha=\varphi_{1}(q, x)$, where $\alpha$ is a pair of symbols $(e, y), e \in E_{1}, y \in Y_{1}$. The next state is $\left(q^{\prime}, t, e, \varepsilon\right)$, where $q^{\prime}=\psi_{1}(q, x)$. It means that the FSM $A 1$ at state $q$ under the input $x$ produces the output $\alpha$, enters the next state $q$ ' and the queue of the channel $\mathrm{C} 12$ is filled by the symbol $e$. Note that $e$ can be equal $\varepsilon$ and, in this case, the next state is a stable state. In similar way, the next state is derived when an external input from $X_{2}$ is submitted.

- Let $g \in G$ be a vertex representing a transient state $\left(q, t, \varepsilon, c_{21}\right)$. Therefore, an external input is absent and a stimulus for a component machine is taken from a queue of the channel C21. Let $c_{21}=c, c \in E_{2}$. Then $g=(q, t, \varepsilon, c)$ and there is an outgoing edge from the state $g$ labeled by pair $c / \alpha$, where $\alpha=\varphi_{1}(q, c)$ is a pair of symbols $(e, y)$. The next state is $\left(q^{\prime}, t, e, \varepsilon\right)$, where $q^{\prime}=\psi_{1}(q, c)$. The same construction is used when channel C12 is not empty.

The whole reachability graph representing the behavior of SCFSM is constructed until all states reachable from the initial state are derived. 
We consider a SCFSM such that any component machine can produce a pair of external and internal outputs as a response to an accepted input. By this reason, given an external input and a stable state, the system may produce a sequence of external outputs in response to the input until the system enters the next stable state. Since we also assume that any internal dialogue is finite, length of this output sequence cannot exceed an appropriate integer $l$. In this case, an external behavior of the system can be described by an FSM where outputs can be sequences of length up to $l$. Thus when a SCFSM has the above properties, in particular it has no live-lock and runs in a slow environment, then its behavior can be described by an FSM that is obtained through the reachability analysis. The composed machine is obtained by hiding all internal actions [2], i.e., symbols of the sets $E_{1}$ and $E_{2}$.

\section{$4 \quad$ Fault Model for Interoperability Testing}

An interoperability test suite is a sequence of external inputs, which are applied to a SUT to verify that its parts can interact with each other as expected. However, most interoperability test suite derivation methods do not return which faults can be detected with a derived test suite. The goal of interoperability testing often is to execute appropriate possible internal interactions only.

Obviously, interoperability testing is concerned only with those failures that occur when protocol entities are interacting. Therefore, it is reasonable to concern about failures occurred through the interaction only, i.e., about a failure when a message produced by one protocol entity has been transformed into another message when reaching another peer entity. In other words, the failure changes a transmitted message. Interoperability testing can be used to detect incompatibilities of protocol implementations such as incompatible options, coding, ect. Note, that compatibility testing can be reduced to media testing, for example the case of incompatibilities of protocol implementations when a message is not accepted by protocol entity due to its format can be considered as the case of a corruption or a loss of a message.

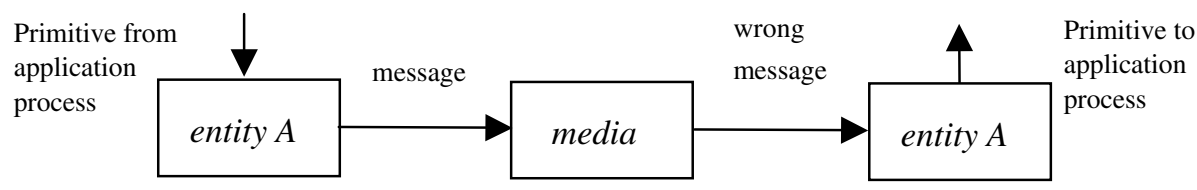

Fig. 2. Communication media with a failure.

Thus we assume that protocol entities are tested separately and found conforming to their specifications, i.e., they are faulty-free. The commutation media including internal interfaces of protocol entities can be faulty; faults of the media correspond to the wrong processing of a given message (Fig. 2). 


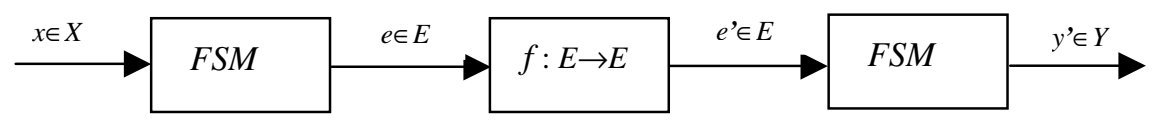

Fig. 3. Media function.

We propose to model a media through which protocol entities interact as a FSM with a single state, i.e., as the mapping $f: E \rightarrow E$, where $E$ is an internal alphabet $\left(E_{1}\right.$ or $E_{2}$ ) (Fig. 3), and consider that the faults of the media FSM do not increase its number of states. Then the function of correct media is the mapping $f_{c}(e)=e$ for any $e \in E$ and the function of faulty media is the mapping $f_{d}(e)=e^{\prime}$ where $e^{\prime} \neq e$ for some $e \in E$. When $e^{\prime} \in E$ then the function of faulty media models the case of a corruption of a message, when $e^{\prime}=\varepsilon$ then we have the case of a loss of a message.

The replacement of a reference message produced by one component FSM by another wrong message can be modeled as the change of one internal output to another internal output. Remember, that an implementation has a transition with an output fault if the output of the transition is different from that of the specification. Thus the faults of the media can be modeled as output faults of a FSM that represents a behavior of a corresponding protocol entity.

Unfortunately, the number of output faults of an FSM is exponential, i.e., can be very large. On the other hand, the results of performed computer experiments [5] show that a test suite complete w.r.t. single output faults of each component machine usually also detects "almost all" multiple output faults. By this reason, in this paper we only consider single output faults. In addition, we assume that at most one component machine can be faulty. Moreover, since interoperability testing is only concerned about interacting between component machines, we only consider so-called internal output faults. In other words, we consider single output faults at the transitions with a non-empty internal output since only such transitions of component machines are responsible for the interaction between component FSMs. Each transition where the empty word is produced as the internal output is assumed to be faulty-free.

We introduce the formal notion of a single internal output fault of a component machine. Let FSM $A 1=\left(Q, X_{1} \cup E_{2}, Y_{1} \times E_{1}, \psi_{1}, \varphi_{1}, q_{0}\right)$ be a specification component machine, while FSM $B 1=\left(Q, X_{1} \cup E_{2}, Y_{1} \times E_{1}, \psi_{1}{ }^{\prime}, \varphi_{1}{ }^{\prime}, q_{0}\right)$ models an implementation of the above specification. We call FSM $A 1$ the reference component while calling FSM $B 1$ an implemented component.

We say that $B 1$ has a single internal output fault if for each pair $(q, x), q \in Q$, $x \in X_{1} \cup E_{2}, \quad \psi_{1}{ }^{\prime}(q, x)=\psi_{1}(q, x)$ and there exists exactly one pair $(q, x)$ such that $\varphi_{1}^{\prime}(q, x)=\left(y, e^{\prime}\right) \neq \varphi_{1}(q, x)=(y, e), e, e^{\prime} \in E_{1}, y \in Y_{1}$. Thus a fault is defined as the replacement of the internal output symbol by a symbol from the same alphabet. A single internal output fault of the component FSM $A 2$ is defined in the same way. 


\section{Interoperability Test Suite Derivation}

\section{$5.1 \quad$ Test Architecture}

Test architecture is an environment where a system under test is tested. Depending on a testing architecture, a system under test has different points of control and observation. It is known that some test suites require proper points of control and observation for protocol entities in order to recognize all the faulty implementations of an appropriate fault domain. It is shown in [5] that a conformance test suite derived by visiting all transitions of each communicating FSM detects all single output faults when there is an observation point at the internal outputs of component machines. In other words, the test suite detects all faulty implementations if we have a possibility to observe internal output reactions of protocol entities. However, sometimes the access to internal interfaces is not granted. In this paper, when deriving a test suite we consider a more difficult option: the access to internal outputs is not granted.

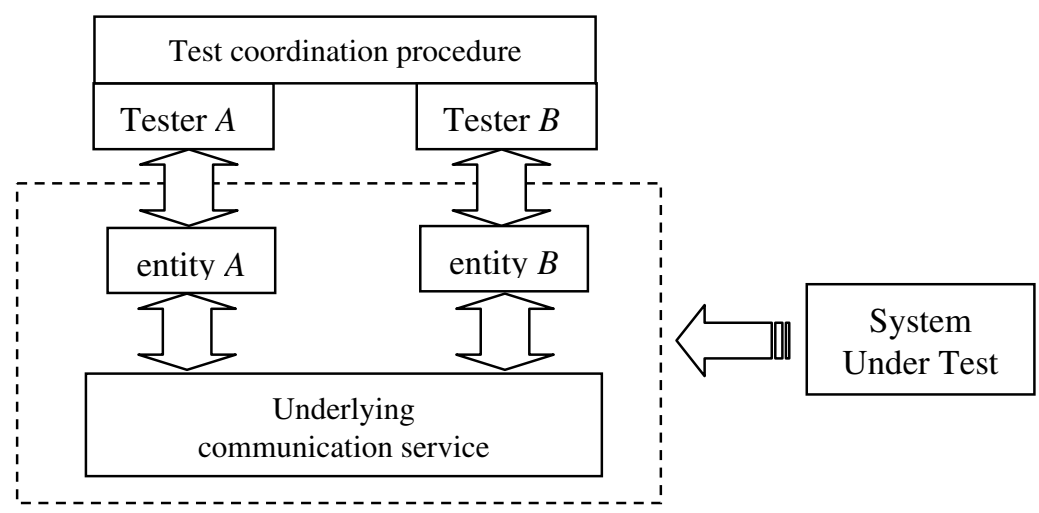

Fig. 4. Test architecture.

Thus we use test architecture in Fig. 4. The SUT is composed of two entities (entity $A$ and entity $B$ ). The upper interface of each entity through which the entity communicates with its upper layer is accessible. The low interface is used by entity to interact with the peer entity in the same layer and it is not accessible. Thus only external output sequences can be observed after applying an external input sequence to the implementation. We also assume that a test synchronization procedure exists between Tester $A$ and Tester $B$.

\subsection{Sketch of a Proposed Method}

As discussed above, an interoperability test suite visits each component transition concerned on interacting of component machines. However, such test suite does not guarantee detecting all output faults since even traversing all transitions of each com- 
ponent machine it is not enough to detect all output faults when no access is granted to internal outputs [5]. Thus the fault coverage of an interoperability test suite remains unknown. Therefore it is required to make the fault coverage analysis of the interoperability test suite as follows: to check if the test suite can detect "almost all" single internal output faults that can occur in traversed transitions. If the test suite does not detect most such faults then additional test cases should be generated. Here we notice that the fault coverage analysis can also be used to detect redundant test sequences, i.e., can be applied to problem of minimizing of test length. In this paper, we don't consider this problem.

Thus we propose the following test generation process. We first derive an interoperability test suite using an existing method. We then evaluate its fault coverage. Finally, we propose how to extend a non-complete test suite if necessary.

In this paper, to derive an interoperability test suite covering appropriate transitions of the specification component machines we are guided by the approach from [8] that is based on a reachability graph of the specification SCFSM. Thus, we assume an initial interoperability test is given and consider two other steps in details. We first evaluate the fault coverage of the test suite w.r.t. single internal output faults of component machines.

\subsection{Fault Coverage Analysis}

Let an interoperability test suite be $I T \subset\left(X_{1} \cup X_{2}\right)^{*}$. For the sake of simplicity, we assume that the test suite does not have sequences, which are proper prefixes of other test cases.

As mentioned above, if a SCFSM has no live-lock and runs in a slow environment then its behavior can be described by a composed machine. Let $\mathfrak{I}$ be the set of composed machines, which can be obtained from the SCFSM when a reference component is replaced by a machine with a single internal output fault. In other words, the set $\mathfrak{I}$ can be derived by explicit simulation of all single internal output faults of each component machine. Let an FSM $N$ denote the composed machine of the specification system. Then the fault coverage analysis can be executed as follows. For each FSM $M$ of set $\mathfrak{I}$ that is not equivalent to FSM $N$ check if an interoperability test suite has a sequence that distinguishes the FSMs $N$ and $M$.

It is known that in general case, the set $\mathfrak{I}$ can have an FSM that is equivalent to FSM $N$. In this case, a behavior of an implemented component with a fault does not influence on a behavior of whole system. Such fault is undetected by any input sequence. We call such fault undetectable [5], since it cannot be detected by any test case.

In general there can exist component transitions that are not reachable from the initial state of the component machine. We further assume when an interoperability test does not traverse an appropriate component transition the transition cannot be traversed at all and do not consider output faults for such transitions.

If for each FSM $M \in \mathfrak{I}$, that is not equivalent to FSM $N$, there exists a sequence in the test suite $I T$ distinguishing $N$ and $M$ then the test suite detects all single internal 
output faults of each component machine and is complete. Otherwise, we are required to extend the test suite. A completeness (the fault coverage) of a test suite is calculated as the ratio $d / f 100 \%$ where $f$ is a number of all detectable faults from the fault domain while $d$ is a number of faults detected with the test suite IT.

We below propose how to perform the fault coverage analysis of a given test suite without explicit enumeration of all faults of the fault domain or at least without explicit enumeration of composed FSMs from set $\mathfrak{I}$.

When no access is granted to internal outputs a single internal fault can be masked by another component machine. By this reason, the absence of a point of observation at internal outputs implies it is not enough to traverse a transition in order to detect a corresponding output fault. However, for some systems, the absence of the points of observation does not influence the observability of a component behavior. For such systems the fault coverage analysis is reduced to checking appropriate properties of component machines. In particular, this is possible if each component machine is an FSM with so-called transparent states.

We say that a state of a FSM is transparent if for each internal input the external parts of all outputs produced at the state are not equal to the empty word and are pairwise different.

The definition of the transparent states leads us to the following proposition.

Proposition 1. If each state of FSMs $A 1$ and $A 2$ is transparent then any interoperability test suite traversing each component transition with a non-empty internal output is complete.

Unfortunately, if the conditions of the proposition are not satisfied then we can say nothing about the completeness of an interoperability test suite.

We further propose the technique for the fault coverage analysis. Given a test case, we derive the reachability graph that besides of reference transitions of appropriate component machine has all transitions with internal output faults. If the corresponding projection of a faulty path is not equal to the expected than the corresponding fault is detected with the test case.

We denote $F(k)=\left\{\left(q, x,\left(e^{\prime}, y\right), q^{\prime}\right): e^{\prime} \neq e\right\}$ the set of all transitions that can be obtained from the transition $k=\left(q, x,(e, y), q^{\prime}\right)$ via a single internal output fault and call a transition $k^{\prime} \in F(k)$ as a faulty (error) transition, while a transition $k$ is called a reference (correct) transition. In similar way, the set of faulty transitions of $A 2$ is defined. Each faulty transition $k^{\prime} \in F(k)$ corresponds to a component machine having a single internal output fault.

Let $G^{\prime}$ be a reachability graph where a reference transition $k$ of an appropriate component machine is replaced by a faulty transition $k^{\prime} \in F(k)$. The graph $G^{\prime}$ is called a reachability graph for faulty transition $k^{\prime}$.

Fault coverage analysis

Input: interoperability test suite $I T$

Output: verdict about test suite completeness

For each sequence $\alpha$ of the set $I T$, do 
1. Construct the path $P(\alpha)$ generated by the sequence $\alpha$ in the reference reachability graph (the reachability graph of specification SCFSM).

2. Obtain the set $R T(\alpha)$ of reference transitions covered by the path $P(\alpha)$.

3. For each reference transition $k \in R T(\alpha)$, derive the set $F(k)$ of faulty transitions.

4. Find a set $W(\alpha)$ as union of sets $F(k)$ over (for all) $k \in R T(\alpha)$.

5. For each faulty transition $k^{\prime} \in W(\alpha)$ do

- construct a deterministic path $P^{\prime}(\alpha)$ generated by the sequence $\alpha$ in the reachability graph for the faulty transition $k$;

- compare the external projection of the path $P(\alpha)$ with that of the path $P^{\prime}(\alpha)$; if they are different then add faulty transition $k$ ' to the set $D(I T)$;

Once a faulty transition is detected by a test sequence, it further is not considered when running the algorithm.

Finally, if the set $D(I T)$ has all faulty internal transitions of each component then the interoperability test suite is complete; otherwise, it is incomplete. Each single internal output fault that is not in the set $D(I T)$ is not detected with the test suite.

Here we notice that when a component machine has undetectable faults then the fault coverage analysis algorithm might produce the verdict about incompleteness though the test suite detects any interaction fault. However, it is well known that the problem to eliminate an undetectable fault has the same complexity as the problem of test derivation for this fault. By this reason, in practical situations, a test engineer usually agrees to have a test suite that detects about $95 \%$ of the faults of the interest.

Example. We now illustrate the proposed algorithm. Consider the system of communicating finite state machines $A 1$ (Fig. 5) and $A 2$ (Fig. 6). Such SCFSM models a behavior of a symmetric communication protocol. Each component machine produces a single meaningful output at a time, i.e., we omit the empty item of the output.

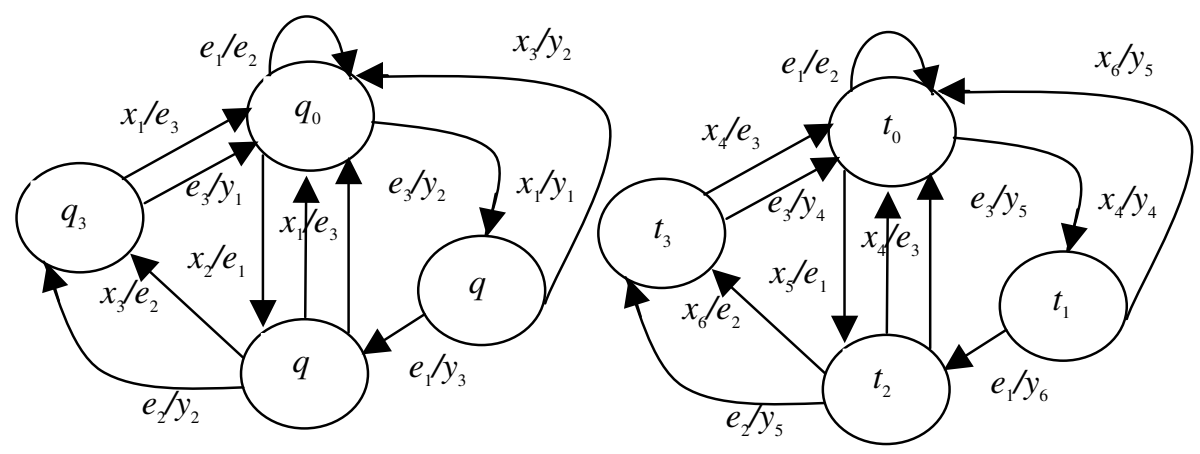

Fig. 5. Component machine $A 1$.

Fig. 6. Component machine $A 2$.

The FSM $A 1$ has input alphabet $X_{1} \cup E_{2}$, where $X_{1}=\left\{x_{1}, x_{2}, x_{3}\right\}, E_{2}=\left\{e_{1}, e_{2}, e_{3}\right\}$, and output alphabet $Y_{1} \times E_{1}$, where $Y_{1}=\left\{y_{1}, y_{2}, y_{3}\right\}, E_{1}=E_{2}$, set of states $Q=\left\{q_{0}, q_{1}, q_{2}, q_{3}\right\}$ with $q_{0}$ as the initial state. The FSM $A 2$ have input alphabet $X_{2} \cup E_{1}$, where $X_{2}=\left\{x_{4}, x_{5}, x_{6}\right\}$, 
and output alphabet $Y_{2} \times E_{2}$, where $Y_{2}=\left\{y_{4}, y_{5}, y_{6}\right\}$, set of states $T=\left\{t_{0}, t_{1}, t_{2}, t_{3}\right\}$ with $t_{0}$ as the initial state. In order to deal with complete FSMs, we augment the machines due to completeness assumption, i.e., for each undefined input the FSM remains at same state while producing external special output " $y_{7}$ " ("null").

The method in [8] returns the interoperability test suite $I T=\left\{\begin{array}{llll}r & x_{1} x_{5}, r x_{2}, r x_{4} x_{2} x_{1}, r\end{array}\right.$ $\left.x_{4} x_{2} x_{3} x_{1}, r x_{4} x_{2} x_{3} x_{4}, r x_{4} x_{2} x_{4}, r x_{4} x_{2} x_{6}, r x_{5}\right\}$. We use the sequence " $x_{2}$ " to illustrate the algorithm.

The path $P\left(x_{2}\right)$ corresponding to the sequence of transitions " $x_{2} / e_{1}, e_{1} / e_{2}, e_{2} / y_{2}$ " covers the set $R T\left(x_{2}\right)=\left\{k_{1}=\left(q_{0}, x_{2}, e_{1}, q_{2}\right), k_{2}=\left(t_{0}, e_{1}, e_{2}, t_{0}\right), k_{3}=\left(q_{2}, e_{2}, y_{2}, q_{3}\right)\right\}$ of reference transitions. Then the sets of faulty internal transitions covered with the path $P\left(x_{2}\right)$ are as follows: $F\left(k_{1}\right)=\left\{\left(q_{0}, x_{2}, e_{2}, q_{2}\right),\left(q_{0}, x_{2}, e_{3}, q_{2}\right)\right\}, F\left(k_{2}\right)=\left\{\left(t_{0}, e_{1}, e_{1}, t_{0}\right),\left(t_{0}, e_{1}, e_{3}, t_{0}\right)\right\}$. We construct the corresponding paths for faulty transitions from $F\left(k_{1}\right) \cup F\left(k_{2}\right)$ (Fig. 7). The only faulty transition $k_{2}{ }^{\prime}=\left(t_{0}, e_{1}, e_{3}, t_{0}\right)$ is undetected by the sequence " $x_{2}$ ", since $Y$-projection of the path $P\left(x_{2}\right)$ coincides with the $Y$-projection of the path $P^{\prime}\left(x_{2}\right)$ corresponding to the faulty transition $k_{2}$ ' and is equal to " $y_{2}$ ". By direct inspection, one can assure the faulty transition $k_{2}$ ' is not detected by any sequence from the test suite. Thus, we conclude that the test suite is not complete.

Finally, the set of undetected faulty transitions is $\left\{\left(t_{0}, e_{1}, e_{3}, t_{0}\right),\left(q_{0}, e_{1}, e_{3}, q_{0}\right),\left(q_{2}\right.\right.$, $\left.\left.x_{1}, e_{2}, q_{0}\right),\left(t_{2}, x_{4}, e_{2}, t_{0}\right),\left(t_{2}, x_{6}, e_{3}, t_{3}\right)\right\}$.

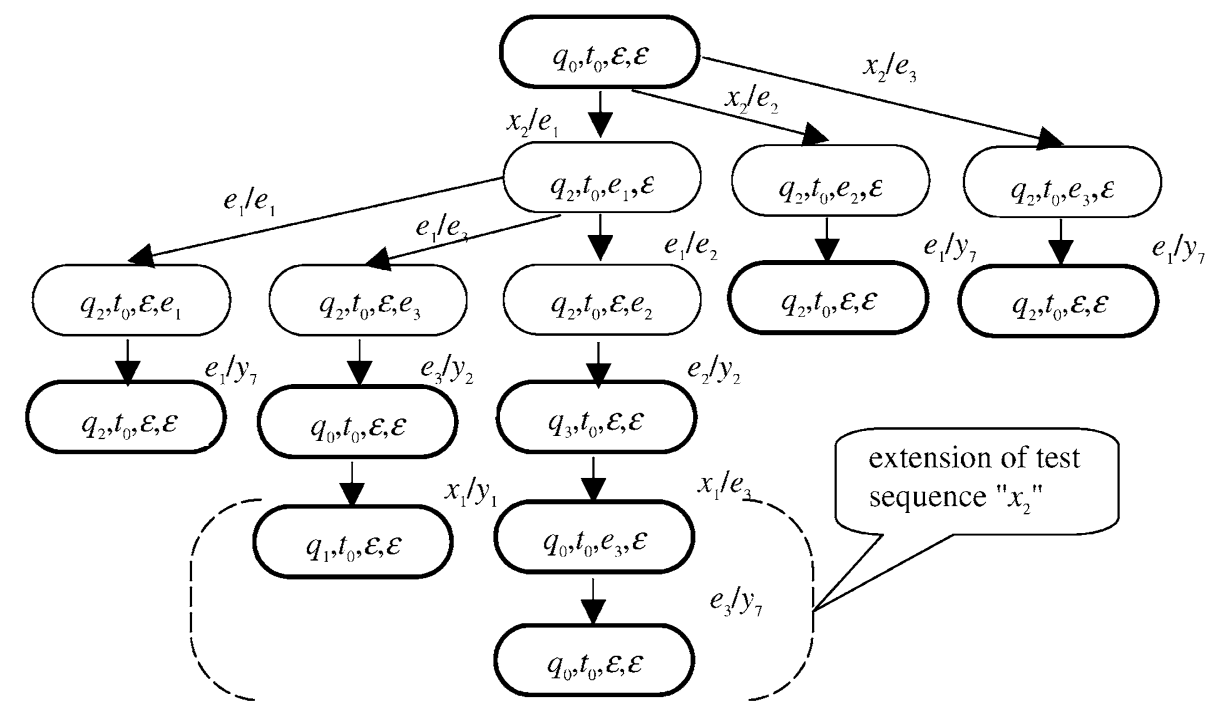

Fig. 7. The reachability graphs corresponding to the test sequence " $x_{2}$ " that has faulty internal transitions.

Thus the general idea of the algorithm is to enumerate all possible faults and to compare reactions of correct and fault systems under the test suite. Here we notice single internal output faults of each component machine can be explicitly enumerated, since their number is proportional to the size of a component machine. Note, that we treat all the output faults corresponded to a given internal transition in paral- 
lel, i.e., we do not derive a faulty composed machine in order to check if the fault is detected with a given test suite.

When a given test suite is not complete we propose a procedure how to augment the test suite in order to detect "almost all" single internal output faults. Note, that if the initial interoperability test suite is complete we do not extend the test suite.

\subsection{Extension of the Test Suite}

Let $P(\alpha)$ and $P^{\prime}(\alpha)$ be the paths generated by the sequence $\alpha$ in the reference reachability graph and in the reachability graph for a faulty transition $k$, respectively.

\section{Random extension algorithm}

Input: interoperability test suite $I T$, length $\max$ of maximum possible extension. Output: extended interoperability test suite $E T$.

For each sequence $\alpha$ in $I T$ do

1. Construct the path $P(\alpha)$.

2. Obtain the set $S(\alpha)$ of undetected faulty transitions covered by the path $P(\alpha)$.

3. For each faulty transition $k^{\prime} \in S(\alpha)$ assign $\beta=\varepsilon$ and do

until length of the sequence $\beta$ is less than $\max$ do

- select randomly symbol $x$ from $\left(X_{1} \cup X_{2}\right)$;

- concatenate the sequence $\beta$ with symbol $x$;

- construct paths $P(\alpha \beta)$ and $P^{\prime}(\alpha \beta)$;

- if the $Y$-projection of the path $P(\alpha \beta)$ is not equal to that of the path $P^{\prime}(\alpha \beta)$ then claim the faulty transition $k$ ' to be detected and replace the sequence $\alpha$ with the sequence $\alpha \beta$ in the test suite $I T$.

4. If the set of undetected transitions is empty then the extended test suite is complete.

If when performing the algorithm a faulty transition is detected by an extended test sequence then further this faulty transition is not considered.

The idea behind the above algorithm is to extend a test sequence symbol by symbol under a reasonable limit. However, the random extension algorithm does not guarantee the construction of a complete test suite. Moreover, in the worst case any random extension of any test sequence can be ineffective. In fact, an extension algorithm when extending a given test suite must try each external input sequence with length up to max. Below we establish an upper bound on length of the sequence max after which the extension becomes useless.

As stated in [14], any two FSMs with $n$ and $m$ states, respectively, can be distinguished by a sequence with length up to $n+m-1$. Since an internal output fault does not increase number of states of a component machine then the specification system and its faulty implementation have up to $n_{1} n_{2}$ stable states where $n_{1}$ and $n_{2}$ are numbers of states of component machines. Therefore, any detectable fault of any component machine is detected by a sequence of length up to $2 n_{1} n_{2}-1$, i.e., the following proposition can be stated. 
Proposition 2. Given a test case $\alpha \in I T$, if a single internal output fault of any component machine is undetected with each sequence $\alpha \beta$, where length of $\beta$ is $\left(2 n_{1} n_{2}-1-\right.$ $|\alpha|)$, then the fault is undetected with any sequence with the prefix $\alpha$.

However, in practical situations the obtained upper bound is too large. By this reason, usually it is enough to check input sequences of length up to $k$ where $k$ is much lower than $\left(2 n_{1} n_{2}-1-|\alpha|\right)$, for example sequences $\beta$ of length up to two.

Thus we propose to extend each test case $\alpha$ with each input sequence of length up to two and to estimate the fault coverage of each extended test case $\alpha \beta$ w.r.t. the set of undetected faulty transitions covered by the path $P(\alpha)$. All faults that remain undetected after this procedure usually are undetectable at all.

Example. Consider the above example. The fault coverage analysis of the test suite $I T=\left\{r \quad x_{1} x_{5}, r x_{2}, r x_{4} x_{2} x_{1}, r x_{4} x_{2} x_{3} x_{1}, r x_{4} x_{2} x_{3} x_{4}, r x_{4} x_{2} x_{4}, r x_{4} x_{2} x_{6}, r x_{5}\right\}$ returns the following result. The faulty transition $\left(t_{0}, e_{1}, e_{3}, t_{0}\right)$ when traversed is undetected by the sequence " $x_{2}$ ". If we replace the test case " $x_{2}$ " with the extended sequence " $x_{2} x_{1}$ " then the faulty transition becomes detected. Fig. 7 shows how the extension algorithm is executed for the sequence " $x_{2}$ ". By direct inspection, one can assure that the faulty transition $\left(q_{0}, e_{1}, e_{3}, q_{0}\right)$ is detected by the sequence " $x_{5} x_{4}$ ", the transition $\left(q_{2}, x_{1}, e_{2}, q_{0}\right)$ by " $x_{4} x_{2} x_{1} x_{4}$ ", the transition $\left(t_{2}, x_{4}, e_{2}, t_{0}\right)$ by " $x_{4} x_{2} x_{4} x_{1}$ ", the transition $\left(t_{2}, x_{6}, e_{3}, t_{3}\right)$ by " $x_{4} x_{2} x_{6} x_{4}$ ". Thus the extended interoperability test suite $E T=\left\{\begin{array}{llllll}r & x_{1} x_{5}, r & x_{2} x_{1}, r & x_{4} x_{2} x_{1} x_{4}, r & x_{4} x_{2} x_{3} x_{1}, r & x_{4} x_{2} x_{3} x_{4}\end{array}\right.$, $\left.r x_{4} x_{2} x_{4} x_{1}, r x_{4} x_{2} x_{6} x_{4}, r x_{5} x_{4}\right\}$ is complete.

\section{Application to TCP}

We consider TCP that $\mathrm{s}$ shown in Appendix 1 where data transfer and a behavior related to a timer are not considered. The interoperability test suite for the TCP derived by the method in [8] is

$\{$ listen_1call_2close_1close_2, listen_1listen_2send_data_1, listen_2call_1close_2close_1, listen_2listen_1send_data_2\}.

The index of an external input or output indicates an appropriate component machine.

Note, that TCP specification is not completely specified. We augment the description of the TCP FSM due to the completeness assumption: for each state and each undefined input at the state the TCP FSM remains at the state while producing external output "null".

In this paper, we use the model of a slow environment, i.e., multiple stimuli and stimuli during transition are not considered. However, the TCP was initially designed to handle situations when both entities send a message at the same time. By this reason, the TCP FSM has unexecuted transitions due to the limitations of the used formal model. Namely, the transitions (SYN_Sent, SYN, SYN_ACK, SYN_Rcvd), (SYN_Rcvd, SYN_ACK, established, Estab), (SYN_Rcvd, close, FIN, FIN_Wait_1), (FIN_Wait_1, FIN, ACK, Closing), (Closing, ACK, closed, Closed) are not covered by the interoperability test suite. Moreover, since there is no state where FIN_ACK 
can be sent the transition (FIN_Wait_1, FIN_ACK, \{ACK, closed $\},$ Closed) cannot be executed too.

Thus each TCP component has nine transitions with an internal output that are reachable from the initial state (see bold edges in the Appendix). The fault coverage analysis returns the following result. The test suite does not detect all single internal output faults in the transition (FIN_Wait1, ACK, NOTHING, FIN_Wait2) and there is an undetected faulty transition (SYN_Sent, SYN_ACK, $\{$ SYN_ACK, established $\}$, Estab). We consider these undetected faults as undetectable since in all cases the correct SCFSM and the SCFSM with the fault have the same final global state. Thus interoperability test suite for the TCP is complete w.r.t. single output faults of the component machines.

\section{Conclusion}

In this paper, we have proposed a fault model for interoperability testing of communication protocols. Interoperability testing can be used to detect incompatibilities of protocol implementations. In one's turn compatibility testing can be reduced to detecting failures when a message sent by one protocol entity is replaced by another message when transmitting to another peer entity. If a joint behavior of protocol entities is described by a system of communicating finite state machines then such failures can be modeled as output faults of component machines. In order to reduce a number of possible faults in the implementation we limit ourselves with single output faults, since it is well known a test suite detecting all single output faults also captures "almost all" multiple faults. Moreover, since interoperability testing is only concerned about interacting between component machines, we are interested only in internal output faults.

Based on the above fault model, we propose an interoperability test suite derivation method with high fault coverage when the access to internal channels is not granted. We establish a sufficient condition when a test suite visiting all transitions, which are responsible for interacting between communicating FSMs, also detects any internal output fault. When the sufficient condition does not hold for component machines, we propose a technique how to augment a given test suite in order to get higher fault coverage. Undetected faults with the augmented test suite can be listed if necessary.

We illustrate the proposed method with the TCP protocol, using the initial interoperability test suite which is derived by another existing method. The performed fault coverage analysis shows that the test suite is complete and does not need to be extended. We are going to adapt the proposed approach to multi stimulus composition of FSMs as well as to interoperability testing based on other formal models of communication protocols. 


\section{References}

1. Bochmann, G., Petrenko, A.: Protocol Testing: Review of Methods and Relevance for Software Testing. Sigsoft Software Engineering Notes, spec. issue., USA (1994) 109-124

2. Luo, G., Bochmann G., Petrenko, A.: Test Selection Based on communicating Nondeterministic Finite-State Machines Using a Generalized Wp-Method. IEEE Transactions on S.E., Vol 20. N 2. (1994) 149-162

3. Petrenko, A., Yevtushenko, N., Bochmann, G., Dssouli, R.: Testing in context: framework and test derivation. Computer communications, Vol. 19 (1996) 1236-1249

4. Lee, D., Sabnani, K., Kristol, D., Paul, S.: Conformance testing of protocols specified as communicating finite state machines - a guided random walk based approach. In IEEE Transactions on Communications, vol. 44, N 5 (1996) 631-640

5. Cavalli, A., Prokopenko, S., Yevtushenko, N.: Fault detection power of a widely used test suite for a system of communicating FSMs. Proceedings of the IFIP TC6/WG6.1 13th Inter. Conf. TestCom2000, (2000) 35-59

6. Petrenko, A., Yevtushenko, N., Bochmann, G.: Fault models for testing in context. Proceedings of the IFIP 1st Joint Inter. Conf. FORTE/PSTV96, (1996) 163-178

7. Rafiq, O., Castanet, R.: From Conformance Testing to Interoperability testing. Proceedings of the 3rd International Workshop on Protocol Test Systems, USA (1990) 371-385

8. Seol, S., Kim, M., Kang, S., Park, Y.: Interoperability Test Suite Derivation for the TCP protocol. Proceedings of the IFIP Joint Inter. Conf. FORTE XII/PSTV XIX, (1999) 357376

9. Kang, S., Shin, J., Kim, M.: Interoperability Test Suite Derivation for Communication Protocols. Computer Networks, 32 (2000) 347-364

10. Viho, C., Barbin, S., Tanguy, L.: Towards a formal framework for interoperability testing. Proceedings of the 21st Inter. Conf. FORTE2001, (2001) 51-68

11. Seol, S., Kim, M., Chanson, S.T.: Interoperability Test Generation for Communication Protocols based on Multiple Stimuli Principle. Proceedings of the IFIP 14th Inter. Conf. TestCom2002, (2002) 151-169

12. Griffeth, N., Hao, R., Lee, D., Sinha R.K.: Integrated system interoperability testing with application to VOIP. Proceedings of IFIP TC6 WG6.1 Joint Inter. Conf. FORTE XIII / PSTV XX, (2000) 69-84

13. Revised Working Draft on "Framework: Formal Methods in Conformance Testing", JTC1/SC21/WG1/Project 54.1 // ISO Interim Meeting / ITU-T on, Paris, (1995)

14. Gill, A.: Introduction to the theory of finite state machine. McGraw-Hill, New York (1962) 207 


\section{Appendix: The FSM of Simplified TCP}

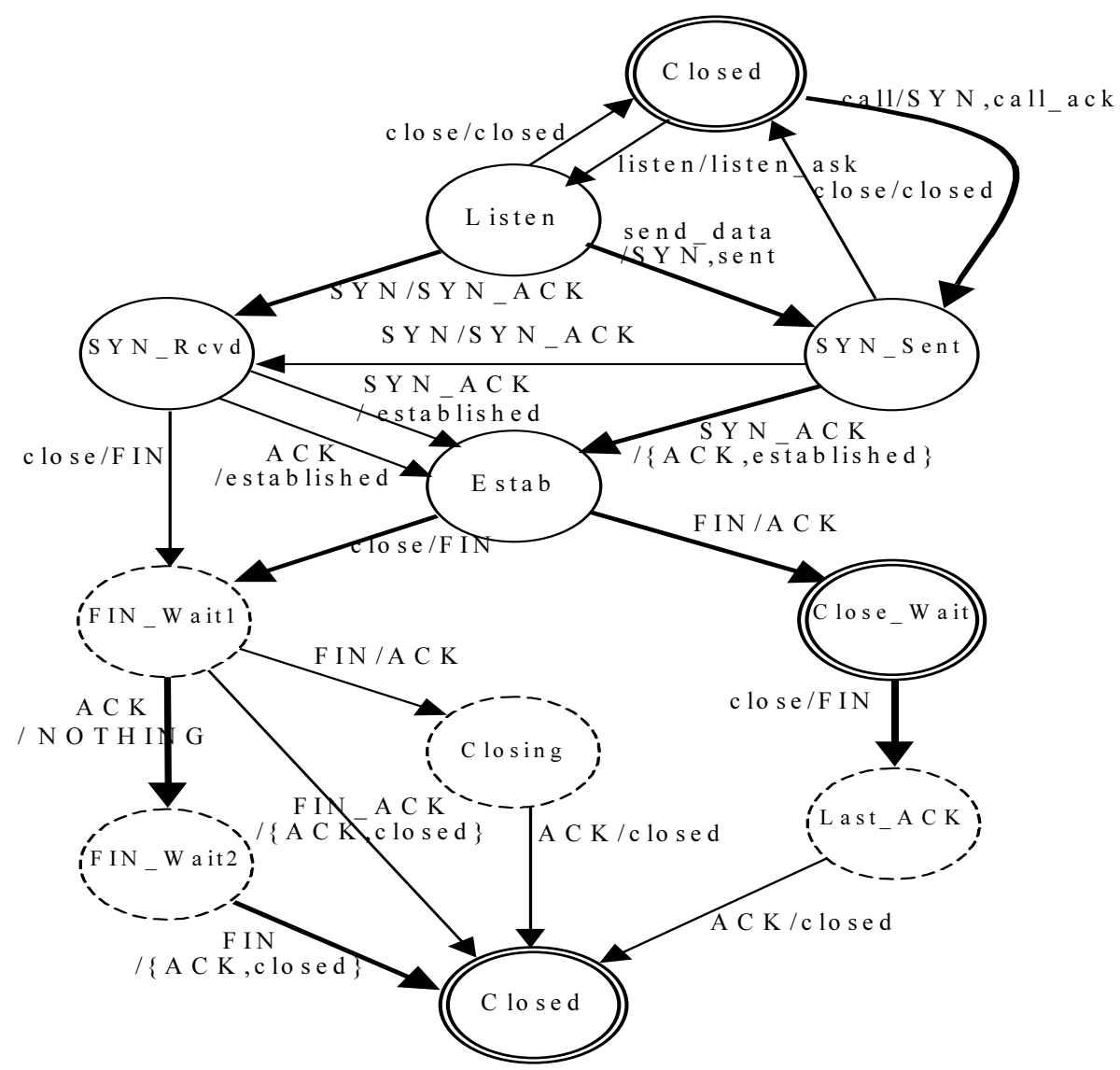

- Input/Output symbols in capital character: messages passing between peerTCPs (internal messages). Input/Output symbols in small character: messages accessing tester through PCOs (external messages). 